\title{
Analizando índices climáticos para predecir la lluvia mensual en una región agrícola de los andes del norte (Caldas, Colombia)
}

\section{Analyzing climate indices to predict the monthly rainfall in an agricultural region of the northern Andes (Caldas, Colombia)}

\author{
Carolina Ramírez C. ${ }^{1,2}$, Jorge J. Vélez U. ${ }^{2}$, Andrés J. Peña Q. ${ }^{3}$
}

\begin{abstract}
Resumen
Varios autores han establecido que diferentes índices, que caracterizan fenómenos oceánicos y atmosféricos en ultramar, tienen relación con el comportamiento del tiempo y el clima en la región andina colombiana. No obstante, la aplicación de este conocimiento es casi nulo y está asociado básicamente a dos situaciones. La primera es la incertidumbre representada por la escasa cantidad de información meteorológica existente para explicar eventos o anomalías climáticas de baja periodicidad. La segunda es la concepción establecida de que el Índice Oceánico El Niño (ONI) es el único índice capaz de describir la variación de los valores de los elementos del clima a escala mensual. Este trabajo trata de reducir las incertidumbres antes mencionadas a través de un estudio comparativo que permite determinar la idoneidad espacio-temporal de diferentes índices y moduladores del clima en una región agrícola del norte de los Andes. Los resultados muestran una mejor correspondencia entre la precipitación de la región y los índices que caracterizan El Niño-Oscilación Sur (ENOS) hasta con tres meses de rezago. No obstante, ONI no es siempre el mejor predictor de la precipitación mensual. Por lo tanto, se podría pensar que algunos índices se podrían utilizar con éxito para pronosticar las lluvias de la mayoría de los meses en las zonas agrícolas de los Andes del norte.
\end{abstract}

Palabras clave: Índices climáticos, lluvias mensuales, agricultura, región Andina.

\begin{abstract}
Several authors have established that different indicators which characterize oceanic and atmospheric phenomena overseas, are related to the behavior of weather and climate in the Colombian Andean region. However, the application of this knowledge is almost the uncertainty represented by the scarce meteorological information available to explain event or climate anomalies of low frequency and the established conception that the El Niño Oceanic Index (ONI) is the only index capable of describing the variation in the values of elements of climate on a monthly scale. This paper aims to reduce the above-mentioned uncertainties by means of a comparative study study to determine the spatiotemporal suitability of different climate indicator and modulators in an agricultural region in the northern Andes. The results show a better correspondence between the region's precipitation and the figures that characterize El Niño-Southern Oscillation (ENSO), which are up to three month behind schedule. However, ONI is not always the best predictor of monthly rainfall. Therefore, one might think that some other indices could be successfully used to forecast rainfall for most months in the northern Andes agricultural areas.
\end{abstract}

Keywords: Climatic indices, monthly rain, agriculture, Andean region.

Recibido el 9 de febrero de 2018, aceptado el 19 de abril de 2018

1 Centro Nacional de Investigaciones de Café, Cenicafé, Km. 4 Vía Antigua Chinchiná-Manizales. Manizales, Caldas. Colombia. E-mail: caramirezcara@unal.edu.co

2 Departamento de Ingeniería Civil, Facultad de Ingeniería y Arquitectura, Universidad Nacional de Colombia, sede Manizales, Cra $27 \mathrm{~N}^{\circ}$ 64-60 Manizales, Caldas. Colombia.

3 Centro de Investigación de la Caña de Azúcar de Colombia, Cenicaña, Km 26 vía Cali-Florida, Valle del Cauca. Colombia. 


\section{Introducción}

En los Andes colombianos la variación en los acumulados mensuales de precipitación se atribuyen al doble paso anual de la Zona de Confluencia Intertropical (ZCIT) (Hastenrath, 2002, 1990, 1967; Portig, 1965; Riehl, 1979; Waylen, Quesada, \& Caviedes, 1996), sistema predominantemente del Este que acarrea gran cantidad de humedad. Aunque la ZCIT puede describir el comportamiento temporal de las lluvias, el agua precipitada total anual está determinada por el efecto de las montañas como barreras al flujo horizontal de aire (Markowski \& Richardson, 2010). En este sentido, en dicha región se pueden encontrar desde zonas secas, con registros medios anuales inferiores a $600 \mathrm{~mm}$, hasta zonas muy húmedas, con precipitaciones medias anuales superiores a los $4500 \mathrm{~mm}$, que comparten el mismo patrón de distribución intra-anual de lluvias.

Dicho patrón consiste en la alternancia de períodos húmedos (abril a mayo y octubre a noviembre) y períodos secos o menos húmedos (enero a febrero y julio a agosto) separados por épocas de transición (Guzman M \& Baldion-R, 1997; Jaramillo \& Chaves, 2000; Leon A., Zea M., \& Eslava R., 2000; Trojer, 1959).

Esta distribución intraanual de la precipitación determinó el desarrollo de calendarios agrícolas que, en el caso de los cultivos semestrales determinaba épocas de siembra durante los períodos lluviosos, lo que garantiza humedad en el suelo y períodos de cosecha durante las épocas secas, asegurando niveles óptimos de humedad al momento de cosechar los granos.
Sin embargo, durante algunos años el patrón pluvial tiende a modificarse y el total anual de lluvias puede variar significativamente con respecto a los valores medios.

Dichos cambios se atribuyen a otro tipo de factores, entre los que se encuentran las anomalías en el patrón de circulación oceánica, las explosiones volcánicas y variaciones a nivel solar, que afectan los patrones de circulación de mediana y gran escala. Según Lau \& Nath (1994) y Webster \& Lukas (1992), en un planeta cuya superficie es mayoritariamente agua, los océanos están en capacidad de modular las condiciones del clima.

A diferencia de lo que ocurre con la ZCIT, que es un sistema que se desplaza siguiendo el movimiento aparente del sol, y la baja presión presente en el mismo afecta las zonas sobre las que transita, el efecto de los fenómenos que se suceden sobre el Océano Pacifico son más complejos.

La complejidad de la interacción entre la superficie del océano y la atmosfera suprayacente radica en que los cambios generados en regiones específicas del Pacífico no solo afectan el clima de la zona sobre la que se presentan, sino que afectan los patrones de circulación de la atmósfera a escala macro y meso.

El cambio en dichos patrones genera variaciones climáticas que dependen de la región analizada, por lo que generalmente se habla de efectos teleconectados. Dos generadores de dicha variabilidad son El Niño Oscilación del Sur (ENOS) (Ropelewski \& Halpert, 1987; Shukla \& Mooley, 1987; Yarnal, 1985) y la Oscilación Decadal del Pacifico (ODP) (Mantua, Hare, 
Zhang, Wallace, \& Francis, 1997; Minobe, 1997). ENOS es un término utilizado para describir un fenómeno de más baja frecuencia que la ZCIT (Diaz \& Markgraf, 1992; Glantz, Katz, \& Nicholls, 1991; Philander, 1989). El Niño es la componente oceánica, relacionada con cambios en la temperatura superficial del mar, mientras que la Oscilación del Sur es la componente atmosférica y está asociada con cambios en la presión atmosférica al sureste y suroeste de la cuenca del Pacifico (Allan, Lindesay, \& Parker, 1996; Philander, 1989; Ropelewski \& Halpert, 1987).

La ODP se identifica como una anomalía de menor frecuencia que ENOS (20 a 30 años), que hace referencia a las variaciones en la temperatura superficial del océano en el noreste y la región tropical, pero que se limita para la región ecuatorial en el Pacífico Oriental, pero que se presenta más intensamente en el Pacífico Norte extratropical (Zhang, Wallace, \& Battisti, 1997).

Aunque las teleconexiones entre la lluvia acumulada a diferentes escalas temporales en diferentes localidades de los andes colombianos y la ODP y ENOS han sido descritas en amplitud y profundidad (Jaramillo R. \& Arcila P., 2009a, 2009b; Montealegre Bocanegra \& Pabón Caicedo, 2000; Poveda \& Mesa, 1996; Poveda et al., 2002; Puertas Orozco \& Carvajal Escobar, 2011; Tootle, Piechota, \& Gutiérrez, 2008; Ruiz \& Pabón, 2013), incluyendo análisis de las interacciones entre las mismas (Verdon \& Franks, 2006), aún no se cuenta con sistemas que permitan hacer una predicción estacional que satisfaga las necesidades de los empresarios que se dedican a la generación de biomasa a partir de energía lumínica.
De hecho, aunque en la actualidad se cuenta con varios índices y variables para caracterizar el efecto de ENOS, la mayor parte de los tomadores de decisiones utilizan el Índice Oceánico El Niño (ONI), por ser el indicador oficial de la condición prevaleciente de ENOS y por su facilidad de interpretación. El ONI se define como la anomalía de la temperatura de la superficie del Océano Pacífico en la región El Niño 3.4 $\left(05^{\circ} \mathrm{S}-05^{\circ} \mathrm{N}, 170^{\circ} \mathrm{W}-120^{\circ} \mathrm{W}\right)$ con respecto a un determinado período de referencia (1971-2010) (Trenberth, 1997; Trenberth \& Stepaniak, 2001; Reynolds, Rayner, Smith, Stokes, \& Wang, 2002; Tootle et al., 2008). No obstante, además del ONI existen otros índices que caracterizan el ENOS. De hecho, algunos índices pueden combinarse para crear índices compuestos, como es el caso de la serie de tiempo Bivariada del ENOS (BEST) (Smith \& Sardeshmukh, 2000). Además de lo anterior, generalmente los estudios no consideran efectos de rezago que pueden hacer que los valores actuales de los índices que describen los procesos de variabilidad climática tengan desajustes temporales.

Debido a esta necesidad por esquemas de predicción del clima que reduzcan el riesgo de que los productores agrícolas sufran pérdidas económicas por efecto del exceso y/o déficit de precipitación, se utilizó datos de clima de la zona montañosa del departamento de Caldas para caracterizar la variación espacial y temporal de la relación existente entre tres indicadores que caracterizan dos generadores de variabilidad climática y la lluvia mensual. Por lo tanto, el objetivo de este estudio es encontrar uno o varios índices que puedan ser utilizados para predecir la lluvia mensual en la zona cafetera de Caldas, Colombia. 


\section{Materiales y métodos}

\section{Características de los datos}

Se utilizaron los datos de precipitación acumulada mensual de 16 estaciones meteorológicas que representan cada una de las zonas climáticas del Departamento de Caldas (figura 1). Las estaciones hacen parte de la Red Meteorológica Convencional administrada por el Departamento de Agroclimatología del Centro Nacional de Investigaciones de Café (CENICAFÉ). La totalidad de estaciones seleccionadas cuentan con 30 años de datos (1984 - 2014). Los acumulados mensuales de precipitación se generaron a partir de series diarias de precipitación que originalmente tenían en promedio $4 \%$ de datos faltantes. Los datos faltantes se generaron a escala diaria usando el Su emulador (Chica Ramírez, Peña Quiñones, Giraldo Jiménez, Obando Bonilla, \& Riaño Herrera, 2014), software que utiliza cadenas de Markov de ordendos para la generación de faltantes. Las series mensuales de los índices que se consideran en este trabajo (ONI, BEST y ODP) se obtuvieron de la base de datos del Climate Prediction Center (CPC) de NOAA. Específicamente para ONI, se utilizó la versión más actual (ERSSTv4). El mapa que describe el área de estudio fue realizado en ArcMap 10.3.1

\section{Caracterización del efecto de los indicadores sobre la lluvia}

Se utilizaron regresiones lineales para caracterizar el efecto de cada uno de los índices sobre el acumulado mensual de lluvia. El grado de asociación lineal entre cada par de variables se determinó con base en el coeficiente de correlación (r) de
Pearson, asimismo se determinó el sentido de la pendiente para establecer si la relación es positiva o negativa. La estimación de las correlaciones se realizó para diferentes rezagos ( 0 a 3 meses), considerando que los indicadores de variabilidad climática describen eventos que anteceden a los eventos de precipitación en la región de estudio.

Para determinar la significancia estadística de las correlaciones se realizó una prueba F. Se consideró que existe relación lineal significativa cuando el nivel crítico p-value fue menor que el nivel de significancia establecido en 5\%. Este análisis permite identificar para cada mes y estación, el indicador que tiene una mejor correlación con la lluvia mensual.

Los análisis estadísticos de este estudio se llevaron a cabo en RStudio 1.0.136 y $\mathrm{R}$ 3.3.3, utilizando el paquete plyr, y por medio del paquete ggplot2 y ggthemes (Wickham, 2009) se construyeron matrices de correlaciones. Dichas matrices permiten describir la relación entre las variables analizadas a nivel espacial y temporal (Wilkinson \& Friendly, 2009).

\section{Caracterización espacial de las correlaciones}

Para facilitar el análisis de los datos, se elaboraron mapas mensuales en los que se asigna a cada estación el nombre del índice que mejor correlación tiene con la lluvia de cada estación. El número que acompaña al índice indica el mes del índice que mejor correlaciona con la lluvia. El análisis de resultados se realizó de acuerdo con las temporadas húmedas y secas en el año. 

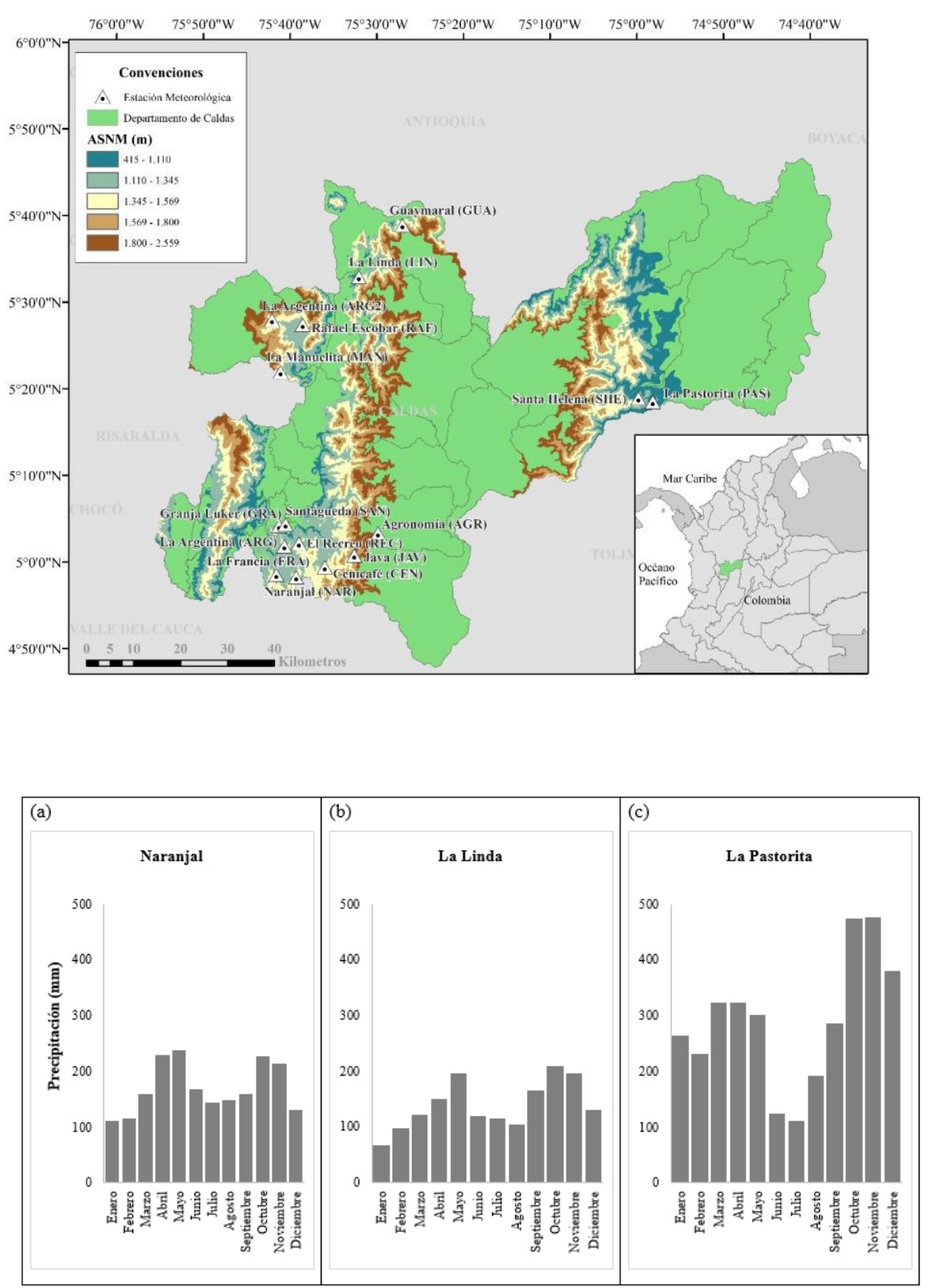

Figura 1. Localización de las estaciones meteorológicas utilizadas en el estudio y precipitación histórica de una estación que representa la zona (a) sur-occidente (b) nor-occidente y (c) oriente del departamento de Caldas. Fuente: Elaboración propia (2018).

Figure 1. Location of meteorological stations used in the study and historical precipitation of a station that represents the area (a) south-west (b) north-west and (c) east of the department of Caldas. Source: Own elaboration (2018). 


\section{Resultados y discusión}

\section{Asociación lineal temporal y espacial entre tres indicadores y la lluvia mensual.}

Correlaciones con el BEST. Los valores del índice BEST están asociados con los valores de precipitación mensual de las localidades. Este índice puede explicar buena parte de la cantidad total de precipitación mensual del trimestre comprendido entre noviembre y enero.

Durante dicha época las relaciones son negativas en general, pero especialmente significativas durante noviembre $\mathrm{y}$ diciembre. Durante el periodo comprendido entre abril y mayo, así como en octubre las correlaciones son bajas, poco significativas y en algunos casos oscilantes entre positivas y negativas dependiendo de la localidad.

La otra época en la que predominan correlaciones significativas es durante el mes de agosto, período en el que las correlaciones son predominantemente negativas. En términos generales los rezagos no determinan incrementos en la significancia de las correlaciones de los meses en los que el índice y la lluvia están poco correlacionados sin aplicar ningún rezago; pero sí permite determinar el comportamiento de la precipitación acumulada con hasta tres meses de anticipación en algunas localidades (figura 2 ).
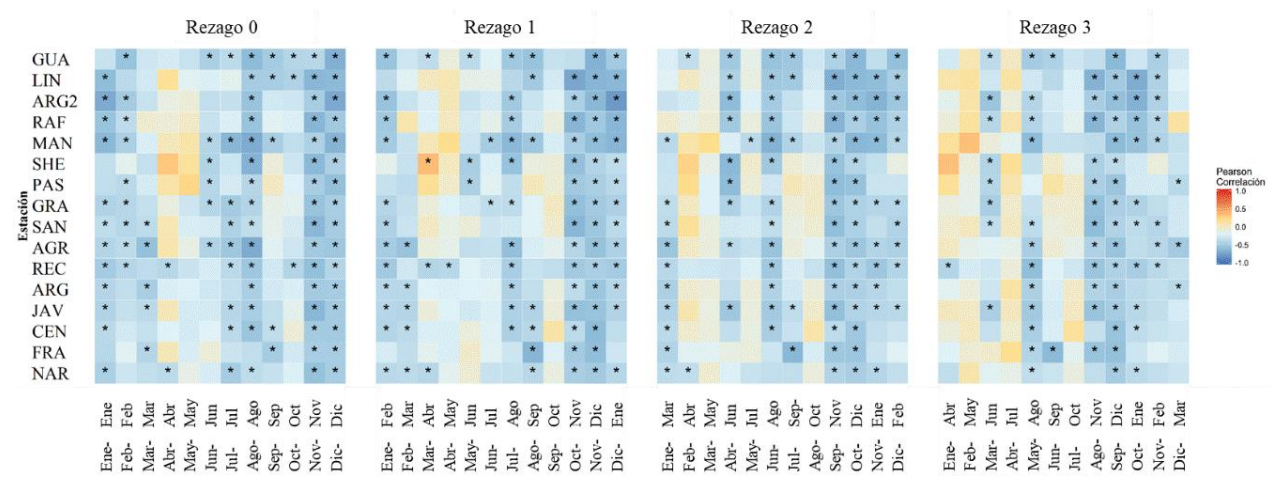

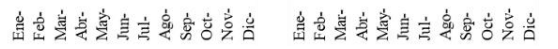

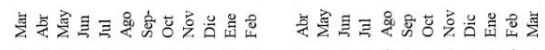

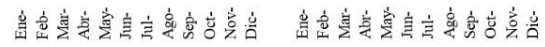

Figura 2. Correlación obtenida entre el índice BEST y la lluvia para cada mes y estación meteorológica. Estaciones ordenadas de sur a norte. (*) Indica significancia estadística al 95\%.

Fuente: Elaboración propia (2018).

Figure 2. Correlation obtained between the BEST index and rainfall for each month and weather station. Stations are arranged from south to north. (*) Indicates statistical significance at $95 \%$.

Source: Own elaboration (2018).

Correlaciones con el ONI. Este índice tiene una relación negativa con la lluvia mensual en general, pero significativa solo en algunas épocas del año. Los valores de correlación de este índice con la lluvia acumulada de los meses de enero, febrero, agosto, noviembre y diciembre, es alta tanto para el análisis con rezago 0 hasta 3 .
Sin embargo, a partir del segundo rezago las correlaciones son más débiles y menos significativas. Se observa también que las correlaciones entre el índice ONI y la lluvia del mes de marzo y junio desde 0 hasta 3 rezagos, presentan correlaciones negativas con significancia estadística. No obstante, existe una diferenciación 
geográfica en dicho comportamiento, es así como las lluvias de las estaciones del sur de Caldas tienen relación significativa con el índice sólo durante marzo, mientras que en el norte de Caldas la significancia se presenta durante el mes de junio.

Particularmente las estaciones ubicadas al oriente de Caldas, especialmente La Pastorita (PAS), presentan una correlación significativa con el ONI para los 4 rezagos analizados en los meses de junio, noviembre y diciembre. En la estación Santa Helena los valores de correlación son positivos y significativos en abril cuando se analizó rezago 2 y 3 , esto indica que cuando la temperatura del Océano Pacífico aumenta con relación a su valor histórico en los meses de enero y febrero, las lluvias en esta estación también aumentan, mientras que cuando la temperatura del Océano disminuye, las lluvias también disminuyen (figura 3).

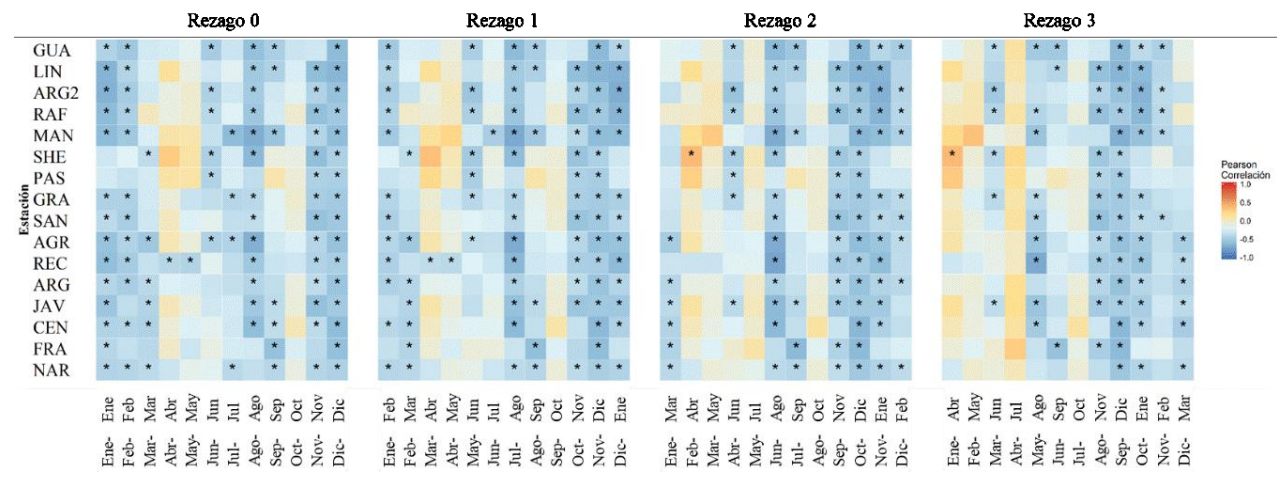

Figura 3. Correlación obtenida entre el índice ONI y la lluvia para cada mes y estación meteorológica. (*) Indica significancia estadística al 95\%. Fuente: Elaboración propia (2018).

Figure 3. Correlation obtained between the ONI index and the rainfall for each month and weather station. (*) Indicates statistical significance at 95\%. Source: Own elaboration (2018). Source: Own elaboration (2018).

Correlaciones con la ODP. La relación entre este índice y la lluvia de Caldas revelan en su mayoría, tanto para el análisis con rezago 0 hasta 3, correlaciones positivas. No obstante, sobresalen correlaciones significativas sólo en algunas estaciones, donde la lluvia depende significativamente de este índice, especialmente durante marzo, agosto, noviembre y diciembre para todos los rezagos. El efecto es más notorio cuando el índice antecede dos meses a la precipitación del mes de diciembre, donde se obtuvo una correlación negativa significativa para el $100 \%$ de las estaciones analizadas.

Es importante estudiar los mecanismos físicos de la asociación en esos meses particularmente. Sobresalen las estaciones La Linda y La Manuelita, ubicadas sobre la cordillera occidental vertiente oriental, las cuales presentan una relación significativa en 4 y 5 meses con la ODP, respectivamente, cuando la correlación se realizó simultánea con la lluvia (figura 4). 

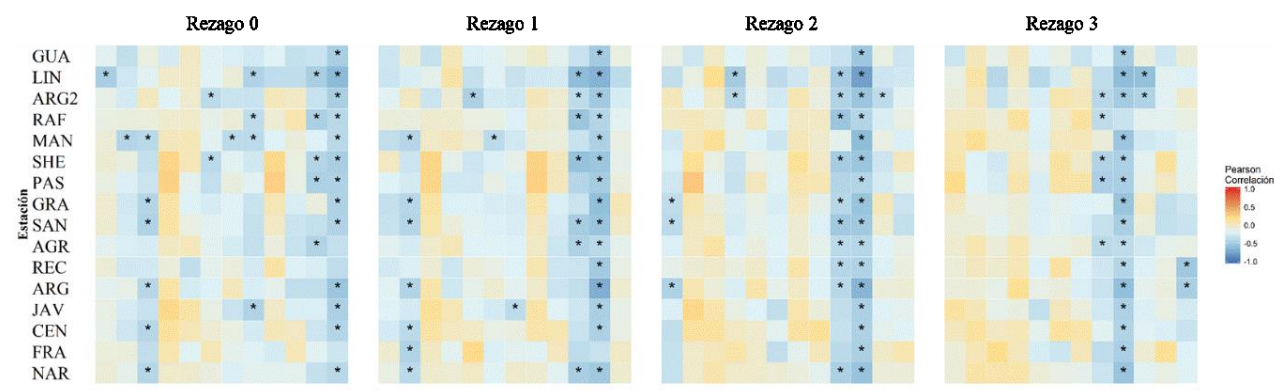

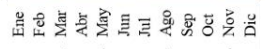

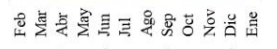

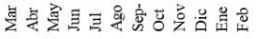

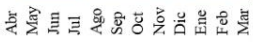

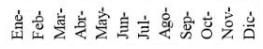

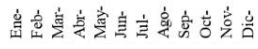

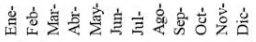

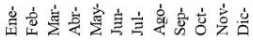

Figura 4. Correlación obtenida entre el índice ODP y la lluvia para cada mes y estación meteorológica. (*) Indica significancia estadística al 95\%. Fuente: Elaboración propia (2018).

Figure 4. Correlation obtained between the ODP index and the rainfall for each month and weather station. (*) Indicates statistical significance at 95\%. Source: Own elaboration (2018).

\section{Índices con mejor correlación para cada mes}

Se destaca el efecto de la ODP sobre la lluvia de algunas estaciones en los meses de marzo, abril, mayo, junio, julio, septiembre, octubre y diciembre (figura 5 y figura 6). Específicamente, en las localidades en las que el índice que caracteriza la ODP tiene las más altas relaciones con la lluvia lo hacen hasta con rezago de tres meses, pero en los meses de junio, julio y diciembre, la relación es mejor con rezago de uno y dos meses. En los meses de diciembre, marzo y octubre, la ODP se correlaciona negativamente con la precipitación de una cantidad considerable de estaciones, lo que representa que la fase positiva de la ODP favorece condiciones más secas de lo normal. Patrones inversos a los anteriores se registraron en el resto de meses, pero las correlaciones no fueron significativas.

No obstante, a pesar de presentar correlaciones significativas con la lluvia, carecen de estabilidad, mostrando una limitada capacidad predictiva sobre la lluvia en esas estaciones. Resultados similares con este índice han sido presentados por diferentes autores (Córdoba, Palomino, Castro, Gámiz, \& Esteban, 2012; Córdoba Machado, Palomino Lemus, \& Esteban Parra, 2014; Rojo, 2011) para correlaciones de ese índice con las precipitaciones y los caudales de los ríos más importantes de Colombia, encontrando una mejor correlación entre las variables hidrológicas y la ODP rezagada dos meses.

Los índices BEST y ONI presentan correlación con las lluvias de todos los meses del año (figura 5 y figura 6). En los meses de abril, mayo, junio, julio, septiembre, octubre y noviembre las correlaciones obtenidas con el índice BEST muestran un mayor ajuste sobre las obtenidas con el índice ONI. La relación entre los índices ENOS y la precipitación acumulada mensual es mejor hasta con tres meses de rezago, pero en mayo, julio y octubre se presenta una correlación directa y débil, dejando ver que estos índices no tienen capacidad predictiva sobre la variabilidad de la precipitación en estos meses para el Departamento de 
Caldas. Estos resultados coinciden con las observaciones realizadas por Ramirez \& Jaramillo (2009), quienes reportaron que los meses históricamente conocidos como de alta precipitación, que tienen influencia de la ZCIT, son los meses que tienen menor efecto de ENOS.

Los resultados de las correlaciones con rezago entre la precipitación acumulada mensual y los índices que caracterizan las condiciones del Océano Pacífico ecuatorial, sonnegativasysignificativas, loquesignifica que cuando la temperatura superficial del mar aumenta, las precipitaciones de las estaciones analizadas tienden a disminuir; lo contrario, es decir mayores precipitaciones, sucederían cuando la temperatura superficial del mar disminuye con respecto al período base. Estos resultados apoyan la idea de Choubin, Khalighi Sigaroodi, Malekian, Ahmad, \& Attarod (2014), en la que se revela que hay una relación más significativa entre las precipitaciones cuando se considera el período de rezago para la predicción, en comparación con la predicción de lluvia cuando la correlación se hace simultáneamente.

Los resultados de las correlaciones entre la lluvia y ENOS, revelan al igual que en otros trabajos (Peña, Ramírez, Bermúdez \& Riaño, 2016; Poveda et al., 2002; Ramirez \& Jaramillo, 2009), la importante influencia de la variabilidad de la temperatura superficial de las aguas del Océano Pacifico tropical sobre la variabilidad de la precipitación en los distintos meses y localidades y el significativo potencial de predicción que tiene el fenómeno ENOS sobre la precipitación en Caldas, Colombia.

Las lluvias del mes de octubre de las estaciones del Departamento de Caldas no presentaron una correlación significativa con ninguno de los índices, a excepción de las estaciones La Linda y Guaymaral ubicadas al Norte del departamento, con una correlación significativa con el índice BEST del mismo mes.

Espacialmente no se logra identificar un patrón geográfico de influencia de cada índice, pero en los meses de abril, mayo y septiembre, que históricamente hacen parte de las temporadas húmedas en el año, los índices BEST y ONI presentan mejores correlaciones con la lluvia de las estaciones del Occidente de Caldas, mientras que las estaciones del Oriente de Caldas correlacionaron mejor con el índice ODP hasta con tres meses de rezago.

Las precipitaciones son moderadamente predecibles basadas en el índice BEST de ENOS, durante enero, febrero, abril, mayo, junio, septiembre y noviembre. Durante enero, febrero y junio, si el valor del índice rezagado es positivo conduciría a que las condiciones fueran más secas, mientras que en los meses de abril, mayo y noviembre, si el índice BEST es negativo, las precipitaciones serían en promedio más altas que lo normal. Éstas anomalías de las precipitaciones relacionadas con ENOS pueden estar moduladas por la ODP y ligadas con la velocidad vertical del viento asociada con la circulación de Walker (Gershunov \& Barnett, 1998; Lyra, Oliveira-Júnior, Gois, Cunha-Zeri, \& Zeri, 2017; Moon, Song, \& Lee, 2015), que crea un cambio significativo en las precipitaciones cuando está en la misma fase con la ODP y un cambio débil cuando está fuera de fase. Según Lyra et al. (2017), cuando el ENOS está fuera de fase con la ODP, se encuentra relacionado con cambios débiles en las condiciones de humedad o déficit. También reporta que en las últimas décadas la ODP se ha vuelto negativa, generando más eventos de $\mathrm{La}$ Niña, promoviendo a que las zonas húmedas seas más húmedas. 

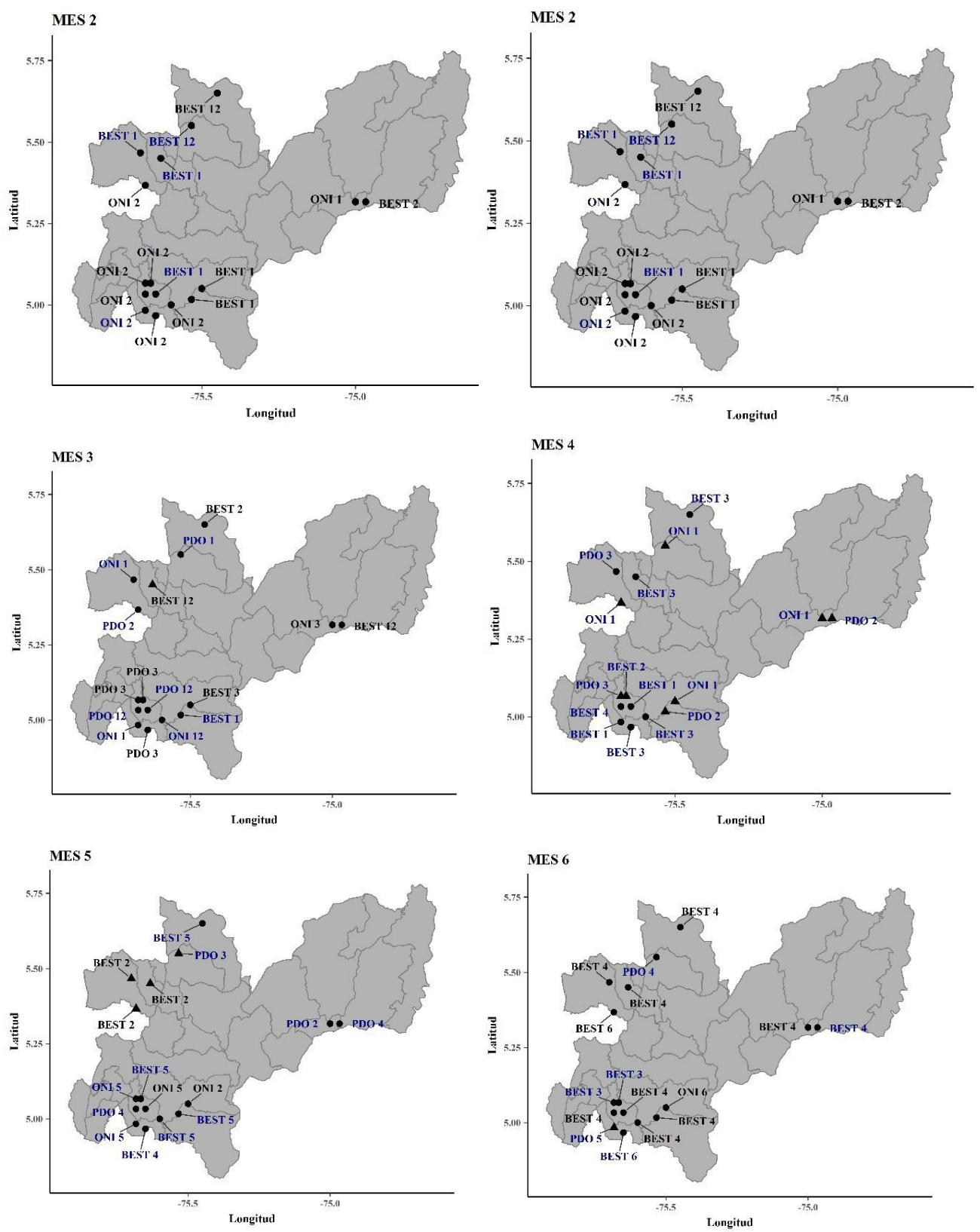

Figura 5. Índice-Mes que mejor correlacionó con los meses de enero, febrero, marzo, abril, mayo y junio. $\bullet$ indica una correlación negativa y $\boldsymbol{\Delta}$ denota una correlación positiva, mientras que los índices que aparecen en texto negro representan correlaciones no significativas y los que aparecen en azul son significativas. Fuente: Elaboración propia (2018).

Figure 5. Index-Month that best correlated with the months of January, February, March, April, May and June. $\bullet$ indicates a negative correlation and $\boldsymbol{\Delta}$ denotes a positive correlation, while the indices that appear in black text represent non-significant correlations and those that appear in blue are significant. Source: Own elaboration (2018). 

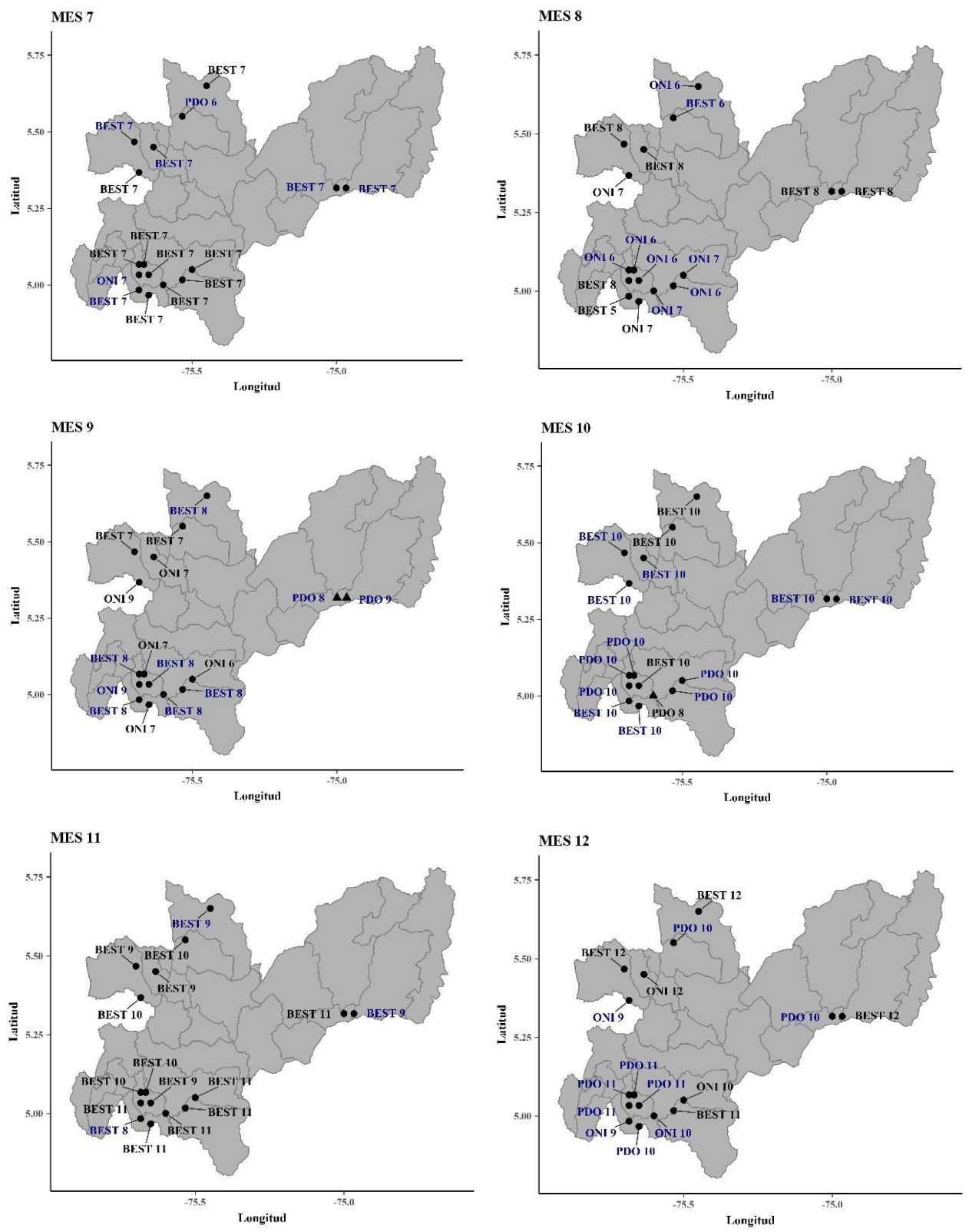

Figura 6. Índice-Mes que mejor correlacionó con los meses de julio, agosto, septiembre, octubre, noviembre y diciembre. $\bullet$ indica una correlación negativa y $\mathbf{\Delta}$ denota una correlación positiva, mientras que los índices que aparecen en texto negro representan correlaciones no significativas y los que aparecen en azul son significativas. Fuente:

Elaboración propia (2018).

Figure 6. Index-Month that best correlated with the months of July, August, September, October, November and December. $\bullet$ indicates a negative correlation and $\boldsymbol{\Delta}$ denotes a positive correlation, while the indices that appear in black text represent non-significant correlations and those that appear in blue are significant. Source: Own elaboration (2018). 


\section{Conclusiones}

En la presente investigación se estudió la relación entre los índices climáticos ONI, BEST y ODP con la lluvia de la zona cafetera de Caldas. Para este fin, se utilizaron 16 estaciones convencionales con 30 años de registros. Se calcularon los coeficientes de correlación entre la precipitación mensual y los índices climáticos con diferentes rezagos, que fueron desde 1 hasta 3 meses. Los resultados de las mejores correlaciones para cada mes, mostraron que el conjunto de los índices tienen una relación inversa con la precipitación, siendo significativas desde 0 hasta 3 meses de rezago, impactando notablemente tanto en las temporadas secas como en las húmedas. En general, las fases frías de los índices tienden a causar mayor ocurrencia de precipitaciones, y las fases calientes de los índices tienden a causar mayor ocurrencia de sequías.

Aunque existe una relación entre los índices que caracterizan ENOS y las precipitaciones en el Departamento de Caldas, no es lo suficientemente fuerte en todos los meses como para predecir de forma consistente y con precisión la precipitación. Sin embargo, los análisis de correlaciones con rezago dejan ver un futuro halagador al utilizar variables más instantáneas que ONI, lográndose utilizar con éxito para pronosticar las lluvias de los meses de enero, febrero, abril, mayo, junio, septiembre y noviembre en Caldas, varios meses antes, ya que la agricultura, especialmente el cultivo del café, depende sensiblemente de las precipitaciones para la realización de labores agrícolas.

$\mathrm{Si}$ se quieren predecir las lluvias, es necesario que la relación entre el índice y la variable tengan un rezago, ya que un rezago cero es anacrónico. El índice BEST por ejemplo, podría usarse junto con un modelo hidrológico para predecir precipitaciones, debido a que éste es un índice cuyo valor se publica mensualmente y la mayoría de resultados mostró mejores correlaciones hasta con 3 meses de retraso. Una alternativa para aprovechar el índice ONI, o cualquier relación simultánea entre uno de los índices con la lluvia, es el uso de las predicciones estacionales para los índices ENOS basados en la Temperatura Superficial del Mar (TSM) hechas por varios centros de pronóstico (IRI). Será necesario actualizar las predicciones a medida que la nueva información de ENOS esté disponible cada quince días, para conducir a mejores predicciones de precipitación en los siguientes meses.

Este trabajó demostró que los distintos índices climáticos utilizados tienen un impacto significativo en las variaciones de precipitación a escala mensual en las 16 estaciones del Departamento de Caldas. Sin embargo, ODP tiene impactos menores sobre las variaciones de precipitación. Los resultados de este estudio proporcionan una base teórica para la previsión de mayores o menores precipitaciones utilizando índices climáticos como predictores en las distintas regiones cafeteras del Departamento de Caldas. Esto puede ayudar a mejorar la mitigación de los riesgos por exceso o déficit hídrico en el suelo.

Las correlaciones entre la lluvia y los índices climáticos están sujetos a una estabilidad fuerte. Este resultado puede ayudar a desarrollar modelos para la predicción de las condiciones húmedas y secas o menos húmedas mediante la adopción de un índice de clima específico como predictor. Por otra parte, los índices climáticos tienden a tener impactos sobre las variaciones mensuales de lluvia hasta después de tres meses, lo que implica que el pronóstico de precipitación mensual sea una tarea desafiante. De 
acuerdo a la investigación, los índices de clima adecuados como predictores para la precipitación mensual son ONI y BEST por presentar mejor significancia estadística.

\section{Agradecimientos}

CENICAFE por suministrar la información necesaria para el desarrollo de esta investigación y el GTA en Ingeniería Hidráulica y Ambiental de la Universidad Nacional de Colombia por apoyar este esfuerzo.

\section{Referencias}

Allan, R., Lindesay, J., \& Parker, D. (1996). El Niño southern oscillation \& climatic variability. CSIRO publishing. ISBN 0643058036

Chica Ramírez, H.A., Peña Quiñones, A.J., Giraldo Jiménez, J.F., Obando Bonilla, D., \& Riaño Herrera, N.M. (2014). SueMulador: herramienta para la simulación de datos faltantes en series climáticas diarias de zonas ecuatoriales. Revista facultad nacional de agronomía Medellín, 67(2), 7.365-7.373. https://doi. org/10.15446/rfnam.v67n2.44179

Choubin, B., Khalighi-Sigaroodi, S., Malekian, A., Ahmad, S., \& Attarod, P. (2014). Drought forecasting in a semi- arid watershed using climate signals: a neurofuzzy modeling approach. Journal of mountain science, 11(6), 1.593-1.605. https://doi.org/10.1007/s11629-014-3020-6

Córdoba, S., Palomino, R., Castro, Y., Gámiz, S., \& Esteban, M.J. (2012). Mecanismos causales de la variabilidad de la precipitación en Colombia. En AEC y GICA (Organizadores), Cambio Climático. Extremos e Impactos. Ponencia llevada a cabo en el VIII Congreso Internacional AEC, Salamanca, España.
Córdoba-Machado, S., Palomino-Lemus, R., \& Esteban-Parra, M. J. (2014). Influencia de la SST y la SLP sobre la variabilidad de la temperatura en Colombia. Revista biodiversidad neotropical, 4(2), 124-132. https://doi.org/10.18636/bioneotropical. v4i2.198

Diaz, H. F. \& Markgraf, V.(1992). El Niño: historical and paleoclimatic aspects of the southern oscillation. Great Britain: Cambridge University Press. ISBN 0-52143042-9

Gershunov, A. \& Barnett, T. P. (1998). Interdecadal modulation of ENOS teleconnections. Bulletin of the american meteorological society, 79(12), 2.715-2.725. https://doi.org/10.1175/1520-0477(1998) 079<2715:imoet>2.0.co;2

Glantz, M.H., Katz, R.W., \& Nicholls, N. (1991). Teleconnections linking worldwide climate anomalies. Cambridge: Cambridge University Press. ISBN 9780521364751

Guzman M., O. \& Baldion-R., J. V.(1997). Regionalización climática de una zona montañosa tropical empleando el análisis multivariado. Revista cenicafé, 48(4), 260274.

Hastenrath, S. L. (1967). Rainfall distribution and regime in Central America. Archiv für meteorologie, geophysik und bioklimatologie, 15(3), 201-241.https://doi. org/10.1007/bf02243853

Hastenrath, S. (1990). Tropical climate prediction: a progress report, 1985-1990. Bulletin of the american meteorological society, 71(6), 819-825. https://doi. org/10.1175/1520-0477(1990)071<0819:TC $\mathrm{PAPR}>2.0 . \mathrm{CO} ; 2$

Hastenrath, S. (2002). The intertropical convergence zone of the eastern Pacific revisited. International journal of 
climatology, 22(3), 347-356. https://doi. org/10.1002/joc.739

Jaramillo R., A. \& Arcila P., J. (2009a). Variabilidad climática en la zona cafetera Colombiana asociada al evento de La Niña y su efecto en la caficultura. (Avance Técnico Cenicafé $\mathrm{N}^{\circ}$ 389). Manizales, Colombia: Cenicafé.

Jaramillo R., A. \& Arcila P., J. (2009b). Variabilidad climática en la zona cafetera Colombiana asociada al evento de El Niño y su efecto en la caficultura. (Avance Técnico Cenicafé $N^{\circ}$ 390). Manizales, Colombia: Cenicafé.

Jaramillo R., A. \& Chaves C., B. (2000). Distribución de la precipitación en Colombia analizada mediante conglomeración estadística. Revista cenicafé, 51(2), 102-113. Recuperado de https://www.cenicafe.org/es/ publications/arc051(02)102-113.pdf

Lau, N.C. \& Nath, M. J. (1994). A modeling study of the relative roles of tropical and extratropical SST anomalies in the variability of the global atmosphereocean system. Journal of climate, 7(8), 1.184-1.207. https://doi.org/10.1175/15200442(1994)007<1184:amsotr>2.0.co;2

Leon A., G.E., Zea M., J. A., \& Eslava R., J.A. (2000). Circulación general del trópico y la zona de confluencia intertropical en Colombia. Meteorología colombiana, 1, 3138. Recuperado de http://ciencias.bogota. unal.edu.co/fileadmin/content/geociencias/ revista_ meteorologia_ colombiana/ numero01/01_05.pdf

Lyra, G. B., Oliveira-Júnior, J. F., Gois, G., Cunha-Zeri, G., \& Zeri, M. (2017). Rainfall variability over Alagoas under the influences of SST anomalies. Meteorology and atmospheric physics, 129(2), 157-171. https://doi.org/10.1007/s00703-016-0461-1
Mantua, N.J., Hare, S.R., Zhang, Y., Wallace, J.M., \& Francis, R.C. (1997). A pacific interdecadal climate oscillation with impacts on salmon production. Bulletin of the american meteorological society, 78(6), 1.069-1.079. https://doi.org/10.1175/15200477(1997)078<1069:apicow>2.0.co;2

Markowski, P. \& Richardson, Y. (2010). Mesoscale meteorology in midlatitudes. Vol. 2. John Wiley y Sons. ISBN-13: 9780470742136

Minobe, S. (1997). A 50-70 year climatic oscillation over the North Pacific and North America. Geophysical research letters, 24(6), 683-686. https://doi.org/10.1029/97gl00504

Montealegre Bocanegra, J. E. \& Pabón Caicedo, J. D. (2000). La variabilidad climática interanual asociada al ciclo El Niño-La NiñaOscilación del sur y su efecto en el patrón pluviométrico de Colombia. Meteorología colombiana, 2, 7-21. Recuperado de http:// ciencias.bogota.unal.edu.co/fileadmin/ content/geociencias/revista_meteorologia colombiana/numero02/02_02.pdf

Moon, J.H., Song, Y.T., \& Lee, H. (2015). PDO and ENOS modulations intensified decadal sea level variability in the tropical Pacific. Journal of geophysical research: oceans, 120(12), 8.229-8.237. https://doi. org/10.1002/2015jc011139

National Centers for Environmental Information. National Oceanic and Atmospheric Administration, NOAA. (n.d). Equatorial pacific sea surface temperatures. Recuperado de https://www.ncdc.noaa.gov/ teleconnections/ENOS/indicators/sst.php

Peña, A. J., Ramírez., C., Bermúdez,

L.N., \& Riaño, N. M. (2016). Rainfall patterns associated with the oceanic niño index in the colombian coffee zone. Journal of agricultural science, 8, 56-63. https://doi. org/10.5539/jas.v8n3p56 
Philander, S.G. (1989). El Niño, La Niña, and the Southern Oscillation. Princeton, New Jersey, Academic Press.

Portig, W.H. (1965). Central American Rainfall. Geographical review, 55(1), 6890. https://doi.org/10.2307/212856

Poveda, G. \& Mesa, Ó.J. (1996). Las fases extremas del fenómeno ENSO (El Niño y La Niña) y su influencia sobre la hidrología de Colombia. Tecnología y ciencias del agua, 11, 21-37.

Poveda, G., Vélez, J., Mesa, O., Hoyos, C., Mejía, F., Barco, O., \& Correa, P. (2002). Influencia de fenómenos macroclimáticos sobre el ciclo anual de la hidrología colombiana: cuantificación lineal, no lineal y percentiles probabilísticos. Meteorología colombiana, 6, 121-130. Recuperado de http://www.matematicas.

unal.edu.co/unciencias/data-file/user_23/ file/METEOROLOGIA/13Poveda $\% 20$ Clima\%20Nuevo.pdf

Puertas Orozco, O.L. \& Carvajal Escobar, Y. (2011). Incidencia de El Niño-Oscilación del sur en la precipitación y la temperatura del aire en Colombia, utilizando el climate explorer. Revista científica ingeniería y desarrollo, 23, 104-118.

Ramirez., V.H. \& Jaramillo, A. (2009). Relación entre el índice oceánico de El Niño y la lluvia en la región andina central de Colombia. Revista cenicafé 60(2), 161 172. Recuperado de https://www.cenicafe.org/es/publications/ar c060\%2802\%29161- 172.pdf

Reynolds, R.W., Rayner, N.A., Smith, T.M., Stokes, D.C., \& Wang, W. (2002). An improved in situ and Satellite SST analysis for climate. Journal of climate, 15(13), 1.609-1.625. https://doi.org/10.1175/15200442(2002)015<1609:aiisas>2.0.co;2
Riehl, H. (1979). Climate and weather in the tropics. London: Academic Press. ISBN 0.12 .588180 .0

Rojo H., J.D. (2011). Desarrollo de un sistema experto para la predicción de caudales medios mensuales en Colombia (Tesis de Magister inédita). Universidad Nacional de Colombia, Sede Medellín.

Ropelewski, C. F. \& Halpert, M. S. (1987). Global and regional scale precipitation patterns associated with the El Niño/southern oscillation. Monthly weather review, 115(8), 1.606-1.626. https://doi.org/10.1175/15200493(1987)115<1606:garspp>2.0.co;2

Ruiz, A.C. \& Pabón, J.D. (2013). Efecto de los fenómenos de El Niño y La Niña en la precipitación y su impacto en la producción agrícola del departamento del Atlántico (Colombia). Cuadernos de geografía: Revista colombiana de geografía, 22(2), 35-54. https://doi.org/10.15446/rcdg.v22n2.3701 5

Shukla, J. \& Mooley, D. A. (1987). Empirical prediction of the summer monsoon rainfall over India. Monthly weather review, 115(3), 695-704. https://doi.org/10.1175/15200493(1987)115<0695: epotsm>2.0.co;2

Smith, C.A. \& Sardeshmukh, P.D. (2000). The effect of ENOS on the intraseasonal variance of surface temperatures in winter. International journal of climatology, 20(13), 1.543-1.557. https://doi.org/10.1002/10970088(20001115)20:13<1543::aidjoc579>3.0.co;2-a

Tootle, G.A., Piechota, T.C., \& Gutiérrez, F. (2008). The relationships between Pacific and Atlantic Ocean sea surface temperatures and Colombian streamflow variability. Journal of hydrology, 349(3- 4), 268-276. https://doi.org/10.1016/j. jhydrol.2007.10.058 
Trenberth, K. E. (1997). The definition of El Niño.Bulletin of the americanmeteorological society, 78(12), 2.771-2.777. https://doi. org/10.1175/1520-0477(1997)078<2771:tdo eno $>2.0 . \mathrm{co} ; 2$

Trenberth, K. E. \& Stepaniak, D. P. (2001). Indices of El Niño evolution. Journal of climate, 14(8), 1.697-1.701. https://doi. org/10.1175/1520-0442(2001)014<1697:LI OENO $>2.0 . \mathrm{CO} ; 2$

Trojer, H. (1959). Fundamentos para una zonificación meteorológica y climatológica del trópico y especialmente de Colombia. Revista cenicafé, 10, 289-373. Recuperado de http://hdl.handle.net/10778/719

Verdon, D. C. \& Franks, S. W. (2006). Longterm behaviour of ENSO: interactions with the PDO over the past 400 years inferred from paleoclimate records. Geophysical research letters, 33(6), L06712. https://doi. org/10.1029/2005gl025052

Waylen, P.R., Quesada, M.E., \& Caviedes, C.N. (1996). Temporal and spatial variability of annual precipitation in Costa Rica and the southern oscillation. International journal of climatology, 16(2), 173-193. https://doi.org/10.1002/ (sici)1097-0088(199602)16:2<173::aidjoc12>3.3.co;2-i
Webster, P.J. \& Lukas, R. (1992). TOGA COARE: The coupled ocean-atmosphere response experiment. Bulletin american meteorological society, 73(9), 1.377-1.416. https://doi.org/10.1175/1520-0477(19 92)073<1377:tctcor $>2.0$. co;2

Wickham, H. (2009). ggplot2: elegant graphics for data analysis. Springer-Verlag New York. https://doi.org/10.1007/978-0387-98141-3

Wilkinson, L. \& Friendly, M. (2009). The history of the cluster heat map. The american statistician, 63(2), 179-184. https://doi.org/10.1198/tas.2009.0033

Yarnal, B. (1985). El Niño/southern oscillation research. The professional geographer, 37(2), 191-193. https://doi. org/10.1111/j.0033-0124.1985.00191.x

Zhang, Y., Wallace, J. M., \& Battisti, D. S. (1997). ENSO-like interdecadal variability: 1900-93. Journal of climate, 10(5), 1.0041.020. https://doi.org/10.1175/1520-0442(19 97)010<1004:eliv>2.0.co;2 